\title{
Economía (étnica) y sociedad. Inmigración y trabajo por cuenta propia en la sociología contemporánea
}

\author{
Alberto RIESCO-SANZ \\ Universidad Complutense de Madrid \\ Sección Departamental de Sociología III \\ albertoriesco@.ucm.es
}

Recibido: 26-10-2012

Aceptado: 06-05-2013

\begin{abstract}
RESUMEN
Este artículo tiene como objetivo realizar un recorrido por las distintas y más significativas formulaciones (y reformulaciones) de la sociología de la empresarialidad étnica contemporánea, analizando su capacidad para explicar sociológicamente el fenómeno de las redes empresariales de las poblaciones étnicamente minoritarias. Nuestra hipótesis de trabajo sostiene que en las principales aproximaciones de la sociología de la empresarialidad étnica, pese a la heterogeneidad de sus propuestas, existirían lugares comunes que van más allá de compartir un mismo objeto formal de estudio. Estos lugares comunes (afinidad, comunitarismo, excepcionalidad étnica) conllevarían importantes limitaciones explicativas (inmediatismo, culturalismo, etnificación) que las distintas reformulaciones efectuadas habrían logrado pulir pero no hacer desaparecer. El artículo concluye reivindicando la necesidad de repensar los términos en los que este campo de estudio ha sido definido y transitado.
\end{abstract}

Palabras clave: economías étnicas, empresarialidad étnica, incrustación, capitalismo, trabajo por cuenta propia

\author{
(Ethnic) Economy and Society. \\ Immigration and Self-employment in Contemporary Sociology
}

\begin{abstract}
The aim of this article is to examine the most significant formulations and reformulations of contemporary sociology of ethnic entrepreneurship, analysing their capacity to provide an explanation in sociological terms for the entrepreneurial networks of minority ethnic groups. Our working hypothesis is that, despite the varying nature of their proposals, the main approaches of the sociology of ethnic entrepreneurship share certain features beyond the fact that they deal with the same area of study. These common features (affinity, communitarism, ethnic exception) involve major explanatory limitations (culturalism, ethnification and a focus on the present or presentism) and, although various reformulations have attempted to counteract them, they have not succeeded in eliminating them completely. The article concludes by insisting on the need for us to rethink the terms in which this field of study is defined and approached.
\end{abstract}

Keywords: ethnic economies; ethnic entrepreneurship; embeddedness; capitalism; self-employment 


\section{REFERENCIA NORMALIZADA}

Riesco-Sanz, A. (2014). "Economía (étnica) y sociedad. Inmigración y trabajo por cuenta propia en la sociología contemporánea". Cuadernos de Relaciones Laborales, Vol. 32, núm. 1, p. 165-190.

SUMARIO: Introducción. 1. Algunos lugares comunes de la sociología de la empresarialidad étnica. 2. Las primeras complicaciones: afinidad, inmediatismo y excepcionalidad étnica. 3. La reformulación de los planteamientos iniciales de la sociología de la empresarialidad étnica. 4. Allegro, ma non troppo: del comunitarismo étnico a los paradigmas accionalistas. 5. A modo de conclusión: el desplazamiento del debate hacia la incrustación de la «economía» en «lo social». 6. Bibliografía.

\section{Introducción}

En las décadas de 1970 y 1980 , en un contexto marcado por el estancamiento económico, el incremento del desempleo y el endurecimiento de las políticas migratorias en la mayoría de los países occidentales, algunos investigadores norteamericanos de lo que se conocerá como la «sociología de la empresarialidad étnica» (Light, 1972; Bonacich, 1973; Bonacich y Modell, 1980; Wilson y Portes, 1980; Portes y Stepick, 1985; Portes y Bach, 1985; Waldinger, 1986a y 1986b; Light y Bonacich, 1988) volvieron a llamar la atención sobre los efectos beneficiosos de los modos de empleo por cuenta propia, esta vez a propósito de las poblaciones minoritarias. Con mayor o menor entusiasmo, muchos de estos planteamientos creyeron ver en los modos de empleo por cuenta propia una herramienta de movilidad social ascendente para las minorías étnicas, una fuente de nuevas y mejores oportunidades de empleo en un contexto económico desfavorable. En definitiva, una vía alternativa de integración que no pasaba necesariamente por la «asimilación» en el mainstream de la sociedad norteamericana. Ahora bien, si esto era así ¿por qué la presencia de la «empresarialidad» era tan desigual entre las minorías discriminadas por motivos étnico-raciales? ¿Por qué no todas tenían el mismo «éxito» emprendedor? ¿Qué factores determinaban que algunos grupos étnicos tuvieran mayor presencia que otros en el trabajo por cuenta propia?

La denominada «sociología de la empresarialidad étnica», una sociología situada a caballo de la sociología económica y de la sociología de las migraciones, trató de responder a estos y otros interrogantes. No lo hizo, sin embargo, de manera homogénea. Los estudios sobre las distintas formas de empresarialidad de las minorías étnicas y/o inmigrantes constituyen, de hecho, un ámbito de las ciencias sociales dinámico y heterogéneo, repleto de no pocas polémicas y diferencias. Polémicas, por ejemplo, en torno a: la propia denominación del fenómeno investigado (minorías intermediarias, economías étnicas, economías de enclave étnico, hegemonía étnica, nichos étnicos, empresarialidad étnica transnacional...); su delimitación empírica (incorporación o no de los miembros de una minoría que trabajan por cuenta ajena en negocios de otros miembros del grupo; demarcación espacial en la que podrían ser correctamente observados estos fenómenos; tipo de actividades y sectores a considerar...); su impacto socioeconómico (actividades marginales o de alternativas de movilidad social ascendente; grado de 
generalización de los posibles efectos beneficiosos derivados...), etc. Como ocurre en otros campos de especialización de la sociología, resulta ilusorio pensar que los estudios sobre la empresarialidad étnica conforman un espacio sin fisuras capaz de aportar respuestas comunes a los interrogantes planteados. Y, sin embargo, esto no ha impedido que sigamos hablando de la existencia de una «sociología de la empresarialidad étnica».

Este artículo tiene como objetivo efectuar un recorrido por las distintas y más significativas formulaciones (y reformulaciones) de la sociología de la empresarialidad étnica contemporánea ${ }^{1}$. El artículo pretende con ello analizar su capacidad para explicar en términos sociológicos el fenómeno de las redes empresariales de las poblaciones étnicamente minoritarias. Nuestra hipótesis de trabajo sostiene que en las principales aproximaciones de la sociología de la empresarialidad étnica, a pesar de la heterogeneidad de sus propuestas, existiría una serie de lugares comunes (no siempre explicitados) que van más allá del hecho de compartir un mismo objeto formal de estudio. Estos lugares comunes iniciales (afinidad, comunitarismo, excepcionalidad étnica) conllevarían algunas importantes limitaciones explicativas (inmediatismo, culturalismo, etnificación) que las distintas reformulaciones de la sociología de la empresarialidad étnica habrían logrado pulir pero no hacer desaparecer. De hecho, como sostendremos en el artículo, los sucesivos desplazamientos de los términos del debate (del comunitarismo étnico al enclaustramiento de la economía en las estructuras sociales), pese a la indudable apertura y avance que han supuesto, volverán a reproducir algunas de estas limitaciones (culturalismo e inmediatismo) al tiempo que incorporarán otras nuevas (sustantivización de la economía, identificación de capitalismo y mercado). Nuestro objetivo, más que negar las aportaciones de este campo de estudio, consiste en reivindicar la necesidad de repensar los términos en los que dicho campo ha sido definido y transitado.

${ }^{1}$ En España, aunque con cierto retraso derivado de la propia especificidad de su dinámica migratoria, en los últimos años también se ha desarrollado con fuerza este ámbito de los estudios migratorios. Entre los trabajos más representativos podrían señalarse, por ejemplo: Aramburu (2002); Arjona (2006); Arjona y Checa (2006); Beltrán, Oso y Ribas (2006); Cavalcanti (2008); López-Castellano, García y Aboussi (2012); López-Sala (2007); Moreras (2005); Oso y Villares (2008); Riesco-Sanz (2003 y 2010); Serra (2006); Solé, Parella y Cavalcanti (2007); Solé, Parella y Alarcón (2009). Aunque con aportaciones valiosas y muchas veces originales, en conjunto estos trabajos no han generado un corpus teórico o una propuesta claramente diferenciable de las grandes aproximaciones que abordaremos en este artículo. Mucho más frecuente ha sido la adaptación y aplicación empírica de unos u otros enfoques a las particularidades del contexto migratorio español; así como, en ocasiones, un diálogo crítico con algunos de los presupuestos de partida de la sociología de la empresarialidad étnica que aquí vamos a considerar. 


\section{Algunos lugares comunes de la sociología de la empresarialidad étnica}

Muchas de las reflexiones pioneras de este ámbito contemporáneo de la sociología partían de una constatación empírica: la capacidad de emprendizaje o empresarialidad en las sociedades modernas no estaba repartida homogéneamente entre la población, sino que determinados segmentos de la misma -«étnicamente» diferenciados- mostraban una mayor especialización en este tipo de actividades económicas, una mayor «inclinación» hacia los modos de empleo por cuenta propia (Riesco, 2010). No se trataba de un hecho baladí: como veremos, dicha constatación implicaría que, en las sociedades capitalistas modernas, el reparto y la asignación social de actividades dentro del conjunto de la población no se efectuaría, principal o exclusivamente, por medio de los mecanismos del mercado. Dicho de otro modo: la lógica «económica» moderna (la dinámica supuestamente «universal», «ciega» y «abstracta» del mercado) no permitiría explicar, por sí sola, la especialización de determinadas poblaciones en el trabajo por cuenta propia pues, de hacerlo, la empresarialidad debería encontrarse presente (o ausente) por igual en todos los segmentos de la población, cosa que no ocurría. En estos planteamientos las economías capitalistas quedaban definidas, ante todo, como economías de mercado. Sin embargo, los mecanismos de mercado parecían incapaces por sí solos de explicar comportamientos económicos como la especialización de ciertas minorías en el trabajo por cuenta propia. Las causas de tales comportamientos habría entonces que buscarlas en las dimensiones y estructuras socioculturales en las que la propia dinámica económica se encontraba inserta, incrustada o enclaustrada, por retomar el término con el que Granovetter (1985) -más que Polanyi- habría inspirado a muchos autores de este ámbito de la sociología.

Cabe señalar, no obstante, que en las primeras y más importantes aproximaciones de la sociología de la empresarialidad étnica (economías étnicas, minorías intermediarias, economías de enclave étnico), tales dimensiones y estructuras socioculturales giraron, en gran medida, en torno a las características y recursos propios de los grupos minoritarios (comunitarismo), situando en ellos -no exclusivamente, pero sí de forma determinante- la clave explicativa del fenómeno investigado (Riesco, 2010). Explicar los diferenciales emprendedores de las minorías étnicas suponía, en último término, dar cuenta de las formas específicas de organización social -incluida la gestión de los negocios- presentes en tales grupos minoritarios (Light, 1972; Bonacich y Modell, 1980; Wilson y Portes, 1980; Portes y Stepick, 1985; Portes y Zhou, 1992). Al margen de cómo se explicase su genealogía, se trataba casi siempre de hacer hincapié en la trama social, cultural e institucional (valores, tejido asociativo, redes, lazos de parentesco, marcos normativos y reglas, etc.) que articulaba internamente a dichas colectividades étnicamente diferenciadas. Dicho de otro modo, sería la presencia o ausencia de este entramado social y de estas formas de vida (que permitían movilizar recursos de todo tipo: financieros, fuerza de trabajo, información, clientes, etc.) lo que determinaba, en última instancia, las posibilidades emprendedoras de unas u otras minorías étnicas. 
¿Y en qué consistían las modalidades de organización social presentes en las minorías étnicas emprendedoras? Básicamente en la existencia de una densa organización comunitaria, trufada de vínculos sociales y articulada en torno a principios «étnicos» y «familiaristas» de pertenencia. Un tipo de organización social -y de gestión de los negocios- en el que la posición ocupada y los recursos disponibles dependerían, en gran medida, del capital simbólico detentado por los miembros de dicho grupo (por sus familias en un sentido extenso). Al margen de cómo hubiera surgido la «etnicidad», la delimitación de la colectividad en términos «étnicos» configuraba una comunidad de orden «moral» que establecía, nítidamente, un adentro y un afuera del grupo. Esto es, establecía una dualización de los marcos normativos presentes en las relaciones sociales y los intercambios, aplicándose unos $\mathrm{u}$ otros en función de que el interlocutor formara o no parte de dicha unidad «moralmente» constituida (Light, 1972; Bonacich, 1975; Bonacich y Modell, 1980; Portes y Stepick, 1993).

Al igual que ocurría en las sociedades tradicionales (precapitalistas), el grueso de las relaciones sociales de estas minorías étnicas parecían regirse por los principios de reciprocidad y obligación típicos de los intercambios en forma de dones y contradones (Mauss, 1950; Polanyi, 1989; Godelier, 1996). Esta solidaridad grupal era consecuencia del sentido de pertenencia a una misma colectividad étnicamente definida (era una solidaridad «étnica») y «moralmente» erigida (constituía una solidaridad «forzosa»). La solidaridad étnica era el resultado de la densidad relacional y de los vínculos sociales de los integrantes del grupo y permitía la movilización de recursos, así como el desarrollo de instituciones y prácticas comunitarias basadas en la reciprocidad y la confianza grupales (Light, 1972; Bonacich y Modell, 1980; Portes y Bach, 1985; Portes y Zhou, 1992; Portes y Stepick, 1993; Portes y Sensenbrenner, 1993). El principio de obligación y la capacidad sancionadora del propio grupo se convertían en mecanismos reguladores más importantes que los vínculos contractuales típicos de las sociedades modernas. Estaríamos, en resumen, ante un modo de organización colectiva en el que el conjunto de las relaciones sociales intracomunitarias -incluidas las económicasquedaban enmarcadas y circunscritas (incrustadas) dentro del marco normativo e institucional que cohesionaba al grupo (Light, 1972; Bonacich, 1973; Bonacich y Modell, 1980; Portes y Sensenbrenner, 1993).

Así pues, podemos decir que entre estas minorías étnicas, lo estrictamente «económico» quedaba supeditado a «lo social». Y era esta supeditación lo que posibilitaba el desarrollo de «alternativas» emprendedoras muy eficaces incluso desde el punto de vista de la lógica económica dominante en las sociedades capitalistas (la lógica «de mercado»). Facilitaba, por ejemplo, la movilización de recursos comunitarios y la acumulación mínima de capital con la que emprender nuevos negocios (mediante asociaciones de créditos rotativos, bancos comunitarios, redes de apoyo basadas en la confianza, etc.). Ponía a disposición de los «emprendedores» una fuerza de trabajo muy movilizada (mediante políticas paternalistas) y de bajo coste, que aceptaba pésimas condiciones de trabajo a cambio del apoyo futuro y la potenciación, por parte de los empleadores, del trabajo 
por cuenta propia entre sus empleados. Hacía posible la externalización (vía su absorción comunitaria) del riesgo innato a las relaciones económicas contemporáneas, al tiempo que reducía la competencia existente entre empresas del mismo grupo étnico (por ejemplo, fijando comunitariamente los precios de venta de los productos o los salarios de los empleados). Lograba la estabilización necesaria del vínculo trabajo-fuerza de trabajo, evitando que los empleados abandonasen sus puestos de trabajo nada más adquirir las competencias o el capital requerido para emplearse por cuenta propia (Light, 1972; Bonacich, 1973 y 1975; Bonacich y Modell, 1980; Portes, 1981; Wilson y Martin, 1982; Portes y Bach, 1985; Light y Gold, 2000).

No eran estas las únicas ventajas: el modelo organizativo de las minorías emprendedoras permitía limitar, además, el impacto negativo que la lógica «económica» moderna tenía sobre la esfera de «lo social» (desigualdad, conflictos, desestructuración, relaciones de explotación entre empleadores y empleados...). El enclaustramiento o incrustación de las relaciones económicas en el entramado social, institucional y normativo del grupo (la «intromisión» de lo comunitario y la imposición de los vínculos solidarios y recíprocos en los intercambios) permitía, en teoría, dotar a sus relaciones sociales de un mayor grado de estabilidad, justicia y equidad, gracias al desplazamiento de los intercambios grupales de la «pura» lógica de mercado («lo económico») hacia la lógica de las convenciones, las normas y las obligaciones («lo social») (Bonacich y Modell, 1980; Portes, 1981; Portes y Stepick, 1985; Portes y Bach, 1985; Portes y Jensen, 1989; Portes y Stepick, 1993).

\section{Las primeras complicaciones: afinidad, inmediatismo y excepcionalidad étnica}

Las propuestas iniciales de la sociología de la empresarialidad étnica que hemos visto subrayaron aspectos importantes para la investigación de las migraciones y las minorías étnicas (heterogeneidad de los modelos de integración; plasticidad de la variable étnica; relevancia de las redes sociales; etc.), al tiempo que llamaban la atención sobre un fenómeno relativamente desconocido como era su capacidad emprendedora. No obstante, estas propuestas movilizaban también -implícita o explícitamente- presupuestos teóricos que limitarían, desde nuestro punto de vista, sus posibilidades explicativas.

Muchas de estas aproximaciones, por ejemplo, abordaron la «enigmática» concentración de ciertas minorías en el trabajo por cuenta propia como el resultado de una confluencia (una afinidad) entre los modos de organización social (formas de vida) de dichas minorías y las características (requisitos) de los sectores económicos en los que se concentraban. Semejante punto de partida dificulta, sin embargo, ir más allá de confirmar lo ya existente y conocido: la presencia, en un momento concreto (conjugado siempre en presente), de un vínculo entre determinados grupos de población étnicamente diferenciados y ciertas formas de empleo basadas en el trabajo por cuenta propia. Supone abordar el fenómeno 
investigado desde cierto inmediatismo, observarlo bajo la coherencia formal propia de todo momento presente (que no puede negar lo que ya es). En estas condiciones, el vínculo entre poblaciones y actividades que estamos investigando adquirirá la apariencia de una estabilidad y una continuidad discutibles en las sociedades modernas (Rolle, 1988 y 2005).

La evacuación de la duración en el análisis sociológico otorgará especial visibilidad a lo idéntico y continuo, quedando la sociedad reducida a orden, concretamente al orden de lo ya existente. La sociología de la empresarialidad étnica, por ejemplo, habría tendido a infravalorar la posibilidad misma de mutación o disolución de la relación existente entre las minorías étnicas y el trabajo por cuenta propia. Una vez constituida históricamente la conexión entre dichas poblaciones y actividades parece que nada pudiera ya perturbar la realización de la «verdadera condición» del asalariado: su transformación en trabajador por cuenta propia $^{2}$. La formulación del problema en términos de afinidad desemboca con facilidad, como vemos, en una argumentación de tipo tautológico: la significativa concentración de determinadas minorías étnicas en actividades por cuenta propia nos permitiría constatar la existencia de una afinidad entre unas y otras. ¿Y a qué se debería esta significativa concentración? ¡Pues a la existencia previa de aquella afinidad!

Parece razonable que planteamientos como estos, que presuponen como estables los vínculos entre las actividades y los sujetos que las desempeñan, pudieran ser oportunos en el caso de las sociedades tradicionales, pero nos parecen difícilmente aplicables allí donde predominan ya las relaciones sociales capitalistas. Por ejemplo, los procesos y mecanismos que, en el siglo XVI, habrían impulsado la especialización en el comercio de una minoría como los judíos (Weber, 1974 y 1993b; Simmel, 1977; Sombart, 2001) ¿serían equiparables a los procesos y mecanismos responsables, en el siglo XXI, de la especialización económica en el comercio de determinados grupos inmigrantes? Es cierto que en ambos casos podemos constatar la existencia de una especialización de ciertas minorías culturales en actividades económicas vinculadas a las formas modernas de empresarialidad. Ahora bien, esta confluencia de hechos empíricos en contextos

\footnotetext{
${ }^{2}$ Una excepción a este respecto lo constituye el trabajo de Bonacich y Modell (1980) sobre las minorías japonesas en Estados Unidos. Ambos autores atienden aquí a su proceso de configuración como minoría intermediaria (dotada de una «economía étnica» con las características ya señaladas), pero también al progresivo debilitamiento de los vínculos comunitarios (y de su red empresarial) conforme se suceden las generaciones en el grupo y se amplían las oportunidades laborales en la sociedad de llegada. También Waldinger (1986a) señalará, aunque sin apenas desarrollarlo, que las minorías «emprendedoras», en el curso de 2 ó 3 generaciones, van progresivamente orientándose hacia el trabajo por cuenta ajena «convencional».
} 
históricos diferenciados no tiene porqué conllevar una coincidencia en las causas que los habrían originado.

Si estos «hechos empíricos» (aparentemente similares) remitiesen ambos a una misma dinámica social, tendríamos que concluir -negando las evidencias apuntadas en no pocos estudios históricos (Braudel, 1985; Hobsbawm, 1988; Polanyi, 1989; Castel, 2004)- que la transformación de las sociedades tradicionales y el tránsito hacia el denominado capitalismo no habría tenido apenas impacto en lo que se refiere a los mecanismos de distribución de las actividades entre las poblaciones. Nos veríamos obligados a sostener que en las sociedades modernas, como ocurría previamente, las poblaciones son asignadas a unas $\mathrm{u}$ otras actividades de forma permanente (en base a principios adscriptivos, por ejemplo). O que la obligatoriedad con la que determinados grupos de población desarrollan ciertas actividades se acompañaría de la prohibición expresa de otras tantas actividades (sobre las cuales podrían pesar restricciones mágico-rituales). En definitiva, tendríamos que afirmar que en las formaciones sociales capitalistas la relación entre poblaciones y actividades se configura como un vínculo duradero, tanto como para conformar una unidad estable, una identidad. Ahora bien, la revolución permanente de los procesos productivos y la potenciación de la movilidad social de las poblaciones ¿no constituyen la base de la fuerza «igualadora» y «subversiva» del capitalismo en comparación al «estancamiento» de las sociedades del Antiguo Régimen? La generalización de una fuerza de trabajo formalmente libre (móvil) y de la competencia entre los distintos capitales ¿no hace saltar por los aires toda pretensión de vincular de forma estable o duradera trabajadores a puestos concretos de trabajo (abriendo así las puertas a la gestión diferenciada de poblaciones y empleos)? (Alaluf, 1986 y 1993)

No son éstas las únicas complicaciones derivadas de los presupuestos de la sociología de la empresarialidad étnica señalados. La afinidad e inmediatismo resultantes de los principios hasta ahora movilizados van a facilitar un repliegue sobre sí mismo del fenómeno objeto de estudio: lo «étnico» de las economías étnicas será explicado a través de las características específicas -«étnicas»- de dichas poblaciones (comunitarismo), facilitando así su desconexión con respecto a otros procesos y dinámicas sociales. Las estructuras ajenas al grupo étnico -una vez infravalorado su dinamismo por la mirada inmediatista- van a funcionar en estos análisis como simples «contenedores» de la acción emprendedora de los sujetos. Concebidas como «contextos» relativamente estables de características comunes para el conjunto de la población, estas «estructuras» poco podrán aportar a la explicación de los diferenciales emprendedores registrados entre minorías. Siguiendo esta lógica, la clave explicativa deberá situarse, como hemos visto, en las dimensiones y características comunitarias de las minorías (formas de vida, identidades, valores, redes y recursos propios, estrategias emprendedoras, instituciones de apoyo, etc.), abriendo así las puertas a los razonamientos en clave de «excepcionalidad étnica».

Muchas de las primeras aproximaciones de la sociología de la empresarialidad étnica habrían favorecido, sin pretenderlo, una etnificación del fenómeno 
investigado (y de su «explicación»), dejando de lado cuánto de estos rasgos supuestamente específicos serían también generalizables a las poblaciones étnicamente mayoritarias. Para la sociología de la empresarialidad étnica la clave del éxito emprendedor de estas minorías residía en sus modos (supuestamente) específicos de «hacer negocios», en la regulación sociocomunitaria de los intercambios económicos. En la medida en que lo comunitario era definido, fundamentalmente, en términos étnicos y era la etnicidad el principio fundamental de articulación y de regulación de los intercambios, se debía hablar de economías étnicas (o de empresarialidad étnica, de economías de enclave étnico, de negocios étnicos...) y no, simplemente, de «economía». Ahora bien, incluso en lo que se refiere estrictamente a la dinámica interna de estos grupos, la consideración de la etnicidad como único o principal elemento articulador de los mismos (tanto como para que sus economías deban ser calificadas de «étnicas» en lugar de, por ejemplo, «masculinas», «cristianas» o «heterosexuales») podría ser juzgada como una hipótesis controvertida.

Algunos de los debates surgidos en el propio seno de la sociología de la empresarialidad étnica parecen sugerir que ni la etnicidad es el único elemento articulador de estas poblaciones, ni el comunitarismo étnico el único instrumento regulador de sus intercambios socioeconómicos. Nos referimos, por ejemplo: i) al debate sobre el acceso limitado de las mujeres a los recursos comunitarios y a la solidaridad étnica como consecuencia de ideologías y prácticas familiaristas tradicionales (Josephides, 1988; Morokvasic, 1988; Dallalfar, 1994; Hillmann, 1999); ii) a los debates sobre la extensión (o no) de los beneficios de las economías étnicas a los paisanos empleados por cuenta ajena en las mismas, sobre la presencia de trabajo no remunerado (de mujeres y jóvenes mayormente) o sobre la proliferación de conflictos laborales entre empleadores y empleados de las mismas minorías étnicas (Sanders y Nee, 1987b; Bonacich, 1988; Zhou y Logan, 1989; Sanders y Nee, 1992; Zhou, 1992; Gilbertson, 1995); iii) al debate sobre la incidencia de otros elementos (religiosos, políticos, generacionales y de género) en la determinación de las fronteras «morales» dentro de las cuales regirían los principios de «solidaridad grupal» (Sanders y Nee, 1987a; Forment, 1989; Pessar, 1995; Nederveen Pieterse, 2003); iv) las polémicas sobre la geometría variable de la propia dimensión «étnica» y su movilización (subetnicidades, etnicidad interna, movilización y desmovilización instrumental de la identidad «étnica») (Bonacich y Modell, 1980; Aldrich y Waldinger, 1990; Der-Martirosian et al., 1993; Kwok Bun y Jin Hui, 1995), etc. Todos estos debates no niegan el impacto de la etnicidad en la dinámica de las redes empresariales de los inmigrantes, pero sí nos invitan a reflexionar críticamente sobre la relevancia explicativa que en ocasiones se le ha otorgado.

Un último ejemplo: algunos investigadores (Gap Min, 1996; Young Kim, 1999), abordando el caso del colectivo coreano en Estados Unidos, llamaron la atención sobre cómo, en un contexto capitalista, era el propio comunitarismo y las obligaciones grupales (en este caso «étnicas») los que obstaculizaban a menudo el crecimiento de sus redes emprendedoras. El estancamiento de los flujos migratorios 
de coreanos a Estados Unidos durante la década de 1980 y el consiguiente descenso de la disponibilidad de su fuerza de trabajo, sumado a su tendencia a abandonar rápidamente los negocios de sus paisanos (conforme adquirían las competencias y el capital necesarios para instalarse por su cuenta), orientó a muchos empresarios coreanos hacia otras fuerzas de trabajo como los mexicanos y los ecuatorianos. Los posibles beneficios de recurrir a los propios paisanos como fuerza de trabajo (la aceptación de bajos salarios y la «docilidad» a cambio de futuras ayudas comunitarias) desaparecían desde el momento en que estos empresarios comprobaban que por el precio de un empleado coreano podían contratar a dos empleados mexicanos (con quienes no tenían además obligación alguna de reciprocidad). En este caso, el éxito económico dependía de «desincrustar» lo máximo posible las relaciones económicas de las estructuras, obligaciones y lógicas comunitarias («étnicas»). ¿Y no era esto precisamente lo que señalaba también Weber (1974; 1993a y 1993b) al considerar improbable -al contrario que Sombart (2001)- que el capitalismo paria (comunitario) pudiera haber actuado como precursor inmediato del capitalismo moderno? Llama la atención que nuestros inmigrantes «emprendedores» parezcan, con frecuencia, más inspirados por los principios de la sociología weberiana que atentos a las reglas comunitaristas del capitalismo paria ensalzadas por las primeras formulaciones de la sociología de la empresarialidad étnica.

\section{La reformulación de los planteamientos iniciales de la sociología de la empresarialidad étnica}

Resulta probablemente demasiado optimista afirmar que las limitaciones de la sociología de la empresarialidad étnica señaladas son, simplemente, un problema del pasado. Sin embargo, tampoco sería justo ignorar los desplazamientos que se han ido produciendo en su interior con el objetivo de superar tales limitaciones. Por ejemplo, Portes y su equipo, referente destacado como hemos visto en este campo de la sociología, contribuyeron a abrir el debate de la empresarialidad étnica al conectar dicho fenómeno con la discusión sobre los usos y fuentes del capital social (Portes y Zhou, 1992; Portes y Sensenbrenner, 1993; Portes, 2000), así como con los debates más generales de la sociología económica contemporánea (Portes, 1995 y 2010).

Lo mismo podría decirse de otras propuestas suyas más recientes como la denominada empresarialidad étnica transnacional. Dicha perspectiva, impulsada junto a otros investigadores como Guarnizo, proponía conectar las iniciativas emprendedoras de los inmigrantes (transnacionalismo desde abajo) con circuitos, redes y dinámicas sociales que trascenderían los límites del Estado-nación, así como con las propias prácticas transnacionales de los gobiernos y las grandes empresas (transnacionalismo desde arriba). Esta tentativa de conectar ambas dimensiones posibilitó una apertura en el modo de abordar las redes emprendedoras inmigrantes (proveedores y circuitos de intercambio implicados, fuentes de 
financiación, mercados disponibles, etc.), reconociendo el impacto en ellas de dinámicas globales que trascenderían no sólo las fronteras espaciales de carácter nacional, sino también las propias fronteras del colectivo inmigrante. Los recursos que hacían posible el éxito de la empresarialidad étnica (y su viabilidad como «alternativa») dejaban de ser, simplemente, recursos locales (o ligados a las sociedades de llegada), debiéndose también considerar aquellos implicados en circuitos transnacionales que conectaban los contextos de partida con los de llegada (además de otros posibles circuitos de intercambio internacionales) (Portes et al., 2002; Guarnizo, 2003).

A estas propuestas de apertura se les podría añadir otras -impulsadas también por destacados investigadores de la sociología de la empresarialidad étnica- como la teoría de recursos de la empresarialidad (Light y Rosenstein, 1995a; Light y Gold, 2000) o la denominada perspectiva interactiva (Waldinger, 1989; Aldrich y Waldinger, 1990; Waldinger, Aldrich y Ward, 1990). Aunque diferenciados, ambos planteamientos habrían apostado también por complejizar el abordaje de los recursos implicados en las iniciativas emprendedoras de las poblaciones minoritarias. A la habitual distinción entre recursos «étnicos» $\mathrm{y}$ «de clase» (Light y Rosenstein, 1995a; Light y Gold, 2000; Aldrich y Waldinger, 1990), se añadían ahora otras como la diferencia entre recursos generales (de uso muy extendido en cualquier negocio -como saber leer-y que estarían disponibles en prácticamente el conjunto de la población) y recursos específicos de la empresarialidad (de aplicación mucho más focalizada -como saber cocinar comida china-y presencia más reducida entre la población) (Light y Rosenstein, 1995a y 1995b). No se trataba, sin embargo, únicamente de poner en juego una mirada más sofisticada en lo referente a las dimensiones, recursos y características constitutivas de las minorías étnicas estudiadas, sino de reconocer también la importancia explicativa de los «contextos» y características de los sectores en los que se concentraban la mayoría de sus negocios.

La perspectiva interactiva, por ejemplo, proponía añadir a las características de las poblaciones implicadas (competencias y expectativas de los inmigrantes, pautas de asentamiento, cultura y formas de vida, redes sociales, estructuras familiares, etc.) el análisis de las estructuras de oportunidad de mercado y de las condiciones de acceso a las mismas, siendo las estrategias de los actores el elemento responsable del ajuste entre unas y otras dimensiones (Waldinger, 1986a y 1986b; Aldrich y Waldinger, 1990; Waldinger et al., 1990). Ya no bastaría, por lo tanto, con atender a las características socioculturales de la población inmigrante, sino que habría que considerar también las circunstancias históricamente contingentes que moldearían las posibilidades emprendedoras disponibles para dichas poblaciones. En particular: i) las condiciones de mercado que posibilitarán o no que los bienes y servicios ofertados vayan más allá del propio colectivo étnico (y que dependerán, en gran medida, de las características del sector considerado); y ii) las vías de acceso a la propiedad o el control de los negocios (que dependerán, sobre todo, del grado de competencia interétnica y de las políticas estatales existentes) (Aldrich y Waldinger, 1990; Waldinger et al., 1990). Algo relativamente similar plantearía, al menos 
formalmente, el enfoque de los recursos de la empresarialidad étnica al hacer hincapié en la necesidad de conectar en el análisis la dimensión de la demanda de empresarialidad por parte de la economía (que haría referencia a las características de los nichos económicos ocupados, la legislación migratoria y laboral, etc.) y la dimensión de la oferta de emprendedores por parte de las poblaciones (que guardaría relación con sus recursos socioculturales, sus redes y capital social, las características demográficas, etc.) (Light y Rosenstein, 1995a y 1995b).

Todas estas nuevas aproximaciones de la sociología de la empresarialidad étnica conllevaron cierto alejamiento de los planteamientos etnicistas previamente presentados. Dejaron también más espacio en sus análisis a dimensiones situadas más allá de los propios colectivos étnicos, matizando así las miradas comunitaristas y solidaristas cuyas limitaciones hemos señalado. Abrieron incluso la posibilidad de poner en relación la empresarialidad de las minorías étnicas con los movimientos y dinámicas de las poblaciones étnicamente mayoritarias. ¿Estaríamos pues ante una formulación del objeto de estudio capaz de escapar del callejón sin salida de la excepcionalidad étnica? En parte creemos que sí, aunque debemos subrayar también que fueron avances tímidos y parciales que no siempre desembocaron en un claro alejamiento de los planteamientos etnicistas, culturalistas y comunitaristas que aquí hemos criticado.

A pesar de la apertura y de la incorporación de nuevas dimensiones al análisis, estas reformulaciones de la sociología de la empresarialidad étnica terminarían también por situar en «lo étnico» (en la movilización de la etnicidad y de los recursos «étnicos», en la gestión comunitaria -étnicamente definida- de los intercambios intragrupales, en el uso de la solidaridad étnica como mecanismo regulador y estabilizador de las relaciones laborales, etc.) el elemento que, en última instancia, proporcionaría a la empresarialidad étnica su especificidad y eficacia. Era la etnicidad la que «explicaría», a fin de cuentas, los diferenciales emprendedores de los inmigrantes y la que nos obligaría a reconocer que no estaríamos ante una «empresarialidad» como las demás. En este sentido, propuestas más recientes como la perspectiva de la incrustación mixta (Kloosterman, 2000; Rath, 2000; Kloosterman y Rath, 2001), aún reconociendo las aportaciones de estos últimos planteamientos, van a criticar su reducción apriorística de la empresarialidad de las minorías étnicas a un fenómeno etnocultural instalado, prácticamente, en un vacío institucional (o la reducción del contexto institucional a las características e instituciones del propio grupo étnico considerado). Van a cuestionar el etnicismo aún presente, a su juicio, en estas reformulaciones (que la empresarialidad de las minorías es necesariamente diferente por el hecho de estar implicadas en ellas poblaciones étnicamente diferenciadas), su culturalismo (que las iniciativas emprendedoras se desarrollan en un entorno institucional idéntico y estable, por lo que los diferenciales emprendedores serán el resultado de las variaciones en los recursos y características grupales) y su funcionalismo (que las actividades desarrolladas por los inmigrantes son, necesariamente, las más adaptadas a sus recursos y características pues, en caso contrario, hubieran desarrollado otras). 
Es posible, por lo tanto, que las formulaciones presentadas en este apartado se hayan quedado cortas en su crítica a las limitaciones de la sociología de la empresarialidad étnica. Sin embargo, si añadimos ahora las propuestas de la incrustación mixta ¿no podríamos dar por consolidado en este ámbito de las ciencias sociales el abandono de los planteamientos etnicistas y comunitaristas? La negativa de esta nueva aproximación a reducir el fenómeno de la incrustación a una simple cuestión de inserción de las actividades de los agentes económicos en redes sociales; su apuesta por una aproximación más «estructural» (inspirada en Polanyi) capaz de considerar el impacto en la empresarialidad de las minorías étnicas de las variaciones -locales, nacionales, internacionales- en los contextos económicos, políticos, sociales e institucionales; o la atención prestada a la configuración de distintos regímenes socioeconómicos, modelos regulatorios y de welfare (Kloosterman et al., 1999; Kloosterman, 2000; Freeman y Ögelman, 2000; Kloosterman y Rath, 2001) ¿no nos coloca, definitivamente, en situación de sortear muchos de los obstáculos hasta ahora señalados? ¿No queda, por fin, formulado el debate de los modos de empleo por cuenta propia de los inmigrantes en términos no etnicistas, ni culturalistas?

\section{Allegro, ma non troppo: del comunitarismo étnico a los paradigmas accionalistas}

En efecto, los debates abiertos por estas reformulaciones impulsaron un desplazamiento y un abandono (aún en ciernes) de muchos de los lugares comunes de la sociología de la empresarialidad étnica que hemos previamente cuestionado, permitiendo así sortear algunos importantes obstáculos que limitaban su capacidad explicativa. Sin embargo, el modo en que los nuevos planteamientos «resolvieron» el debate sirvió también para perpetuar algunos viejos problemas, al tiempo que incorporaba otros nuevos.

Como señalamos, las aproximaciones tradicionales de la sociología de la empresarialidad étnica habían tendido a «descontextualizar» el fenómeno investigado al privilegiar en su abordaje las dinámicas, características y procesos constitutivos de los grupos étnicos. Para las nuevas aproximaciones el desafío consistía en restituir la complejidad. ¿Cómo? Básicamente añadiendo más dimensiones al análisis, atendiendo al impacto de otras esferas implicadas y hasta ahora ignoradas. Es así como, gracias a esta mirada ahora mucho más perspicaz del investigador, «descubríamos» la existencia de una empresarialidad étnica «multidimensional» que podía basarse en la propiedad étnica de sus negocios pero también en el control étnico de un sector de actividad (Light y Gold, 2000), que podía actuar en mercados de características variables (locales, nacionales o internacionales; orientados o no a clientelas «étnicas», etc.) (Aldrich y Waldinger, 1990; Jones et al., 2000) y poner en juego recursos no sólo étnicos, sino también de clase (Light y Rosenstein 1995a), nacionales y transnacionales (Portes et al., 2002), generales y específicos (Light y Rosenstein, 1995b). Una empresarialidad étnica, en 
definitiva, contextualizada y condicionada (incrustada) en distintos modelos regulatorios e institucionales articulados a distintas escalas espaciales (locales, regionales, nacionales, internacionales) (Rath, 2000; Rath y Oliveira, 2008).

Habríamos añadido así nuevos e importantes elementos a nuestro análisis, «descubierto» nuevas piezas del puzzle social. Tendríamos ahora una «representación» más completa de la complejidad implicada en los modos de empleo por cuenta propia de los inmigrantes. Sin embargo ¿puede una descripción suplir una explicación? La superposición de elementos y la configuración de laboriosas tipologías no tienen porqué añadir automáticamente complejidad a nuestros análisis. Además, el establecimiento por parte del investigador de una tipología presupone la existencia de algún tipo de vínculo entre los elementos que la componen. Dichas tipologías, más que convergencias y cristalizaciones de la realidad social que el investigador tenaz no tendría más que «descubrir» y «descifrar», conllevan, en definitiva, la existencia de un modelo teórico. No obstante, la sociología de la empresarialidad étnica ha tendido a no explicitar los presupuestos teóricos manejados en la elaboración de estas tipologías, ni los criterios por medio de los cuáles deberíamos considerar que los elementos que la componen están efectivamente interconectados. Más allá de las categorías incorporadas a la tipología, poco sabemos de las relaciones que existirían y articularían unos y otros elementos, unas y otras esferas sociales.

El principal problema que aquí vemos no radica, sin embargo, en la tendencia de la sociología de la empresarialidad étnica a formular tipologías de carácter descriptivo, sino en el hecho de que, una vez constituida, la descripción tipológica adquiere el rango de «modelo explicativo» aplicable, una y otra vez, a la realidad social. Una realidad social que, observada desde este «modelo», queda definida y delimitada por aquello que previamente había sido enunciado y considerado en la tipología (en teoría meramente descriptiva de lo social): la descripción se transforma en una prescripción. El proceder tipológico (en absoluto exclusivo de la sociología de la empresarialidad étnica) nos coloca nuevamente ante esa sociología instantánea e inmediatista que hemos criticado. Una sociología volcada en confirmar lo que ya sabemos, en constatar la inevitabilidad (la justicia y/o funcionalidad) del momento presente, de aquello que perdura y parece estable. Una sociología de la reproducción social de lo idéntico que hace pasar una descripción por una explicación. Desde nuestro punto de vista, la solución a los problemas señalados no pasa necesariamente por añadir nuevas capas y dimensiones al fenómeno investigado (a modo de «contexto»). No se trata necesariamente de hacer más, sino hacer de otro modo: para empezar, no renunciar a reconstruir sociológicamente el objeto investigado, ni conformarnos con describir la coherencia y el equilibrio aparentes del instante presente, un presente cuyas características parecerían evidentes y no consecuencia del dispositivo de observación desplegado por los investigadores.

No es el único viejo problema que ha persistido en las nuevas aproximaciones de la sociología de la empresarialidad étnica: la apuesta por el "agencialismo" las estrategias y recursos de los «actores» de algunas de ellas (por ejemplo, las 
mencionadas perspectiva interactiva o la teoría de recursos de la empresarialidad) a la hora de explicar los modos de empleo por cuenta propia de las poblaciones minoritarias, nos sitúan nuevamente en la senda del comunitarismo y culturalismo que previamente habíamos cuestionado. Estos planteamientos accionalistas o agencialistas son, a nuestro juicio, la consecuencia lógica de la sociología inmediatista e instantánea antes mencionada. Cuando el objeto de estudio es situado al margen de toda duración y procesualidad (primando en él la coherencia, el equilibrio y la identidad propios del instante) ¿qué otra cosa podría explicar mejor el cambio observado si no la acción de los actores y sus relaciones de poder? ¿Quién mejor que ellos podrá responsabilizarse de los equilibrios del presente o de aquellos aún por llegar?

Este tipo de razonamientos nos colocan ante algunas dificultades: los actores con sus intervenciones y acciones permitirían, supuestamente, dar cuenta de la configuración y dinámica de la sociedad, pero ¿cómo habrían surgido tales sujetos sociales? ¿Son sujetos autoproducidos? ¿O acaso se limitan a actualizar posiciones existentes desde siempre en la sociedad? Ayer esclavos, siervos y proletarios, hoy precarios, mujeres o inmigrantes. Cambiarían así los nombres pero no el fundamento que parecería estar detrás de cualquier formación social: la relación desigual de poder que enfrenta a dominantes y dominados, mayorías y minorías, hegemónicos y subordinados. De ser así, ¿será verdad entonces que la historia de la humanidad es la historia de la lucha de clases? Este tipo de postulados puede ser políticamente movilizador, pero insostenible sociológicamente hablando. Sin embargo, no se afirma algo muy diferente cuando sostenemos que, en las sociedades capitalistas contemporáneas, son los actores y sus acciones, su confrontación continua en heterogéneas luchas de poder, los responsables últimos de la dinámica social, aquellos que garantizan su equilibrio y ajuste. De manera cuestionable, la estructura social queda así reducida a un problema de interacción entre personas o grupos, un problema de relaciones de poder presentes en todas las dimensiones imaginables de la sociedad (Alaluf, 1993). Los modos de dominación característicos de las sociedades modernas pasan a ser caracterizados como modos de dominación inmediatos y antropomórficos (de un grupo sobre otro), en vez de mediados, impersonales y abstractos (Postone, 2006). Paralelamente, la dinámica social, el cambio histórico, quedan ahora reducidos a una sucesión de estados de equilibrio, una repetición de instantes cuyo único hilo conductor posible lo constituirían los acontecimientos coyunturales derivados de las relaciones de fuerza existentes, desde la más remota Antigüedad, entre diferentes grupos sociales (dominantes y dominados).

Para terminar detengámonos brevemente en el principio de no determinación inscrito en este tipo de paradigma accionalista. Según dicho paradigma la acción no sería un resorte automático externamente inducido, ni una conducta ajena a la voluntad (pueden serlo sus consecuencias, pero no la motivación primera que la desencadena) (Pizarro, 1.979). La acción sería incompatible con la determinación pues constituye y es constituida por un principio de libertad. Así pues, como grandes clásicos de la teoría social se han encargado de subrayar (Weber entre 
otros), la acción tiene que estar orientada por un sentido, es más, debe constituir la externalización por parte del sujeto de un sentido subjetivo (Weber, 1993b). Dicho sentido exteriorizado por medio de la acción estaría conformado por una serie de representaciones (de objetos físicos, sociales y culturales, así como del propio sujeto de la acción). ¿Y dónde se ubicaría el origen de estas representaciones compartidas? La respuesta para el accionalismo no puede ser otra que la «cultura» (interiorizada individualmente vía la «socialización») (Pizarro, 1979). De forma que, paradójicamente, volvemos al punto de partida y nos topamos nuevamente con los valores, las normas, los contratos, las convenciones, los usos y las formas de vida tan recurrentes en las formulaciones iniciales de la sociología de la empresarialidad étnica y cuyas limitaciones ya hemos señalado. Si al comienzo fue la acción, al final, será la repetición y reproducción social de lo idéntico.

\section{A modo de conclusión: el desplazamiento del debate hacia la incrustación de la «economía» en «lo social»}

Las reformulaciones y aproximaciones que acabamos de señalar no evitaron la persistencia de algunas importantes limitaciones iniciales de la sociología de la empresarialidad étnica. No obstante, el alejamiento con respecto a sus primeras formulaciones permitieron un desplazamiento del debate hacia los fenómenos generales de la incrustación de la economía en las estructuras sociales. Dicho de otro modo, orientaron la discusión de manera más explícita hacia el debate sobre la relación entre economía y sociedad, así como sobre las características constitutivas y la dinámica de las formaciones capitalistas contemporáneas. Un debate éste ahora más abiertamente abordado aunque presente de forma implícita desde las primeras formulaciones de la sociología de la empresarialidad étnica.

A grandes rasgos podríamos decir que muchas de las formulaciones de la sociología de la empresarialidad étnica parten de una caracterización de la economía en las sociedades modernas similar a la que podemos encontrar en la sociología económica de Polanyi (1989). Este autor sostiene que las economías de estas sociedades (las sociedades capitalistas) estarían basadas en el principio de mercado, es decir, que eran sociedades que garantizaban la producción y distribución de los bienes y servicios por medio del mercado. Dicho mercado sería concebido, además, como un mecanismo autorregulador: la producción y distribución de bienes y servicios, así como la asignación de recursos para satisfacer tales funciones, quedarían determinadas por los precios del mercado, precios cuyas variaciones deberían ser el reflejo, únicamente, de los cambios en la oferta y la demanda. Desde este punto de vista, la economía de mercado aspiraría a poder autorregularse (y explicarse) al margen de cualquier interferencia externa. Para los defensores del paradigma de mercado criticados por Polanyi, la única intervención posible y legítima por parte del Estado (o de otras instituciones y esferas sociales) sería aquella encaminada a garantizar el libre desarrollo de los mecanismos de mercado y, en definitiva, las reglas del juego económico. 
Además, en relación a estas sociedades con economías de mercado autorregulados, Polanyi (1989) va a destacar como rasgo característico la progresiva emergencia de una institución (el mercado) independiente y autónoma del resto de la sociedad. La sociedad quedaba así dividida en distintas esferas sustantivas (por ejemplo, lo económico versus lo político y/o lo social). La economía, en las sociedades capitalistas contemporáneas, se configuraba como una realidad sustantiva y autónoma identificable con el mercado. Una institución de finalidad y principios exclusivamente económicos (autorregulada y con una inclinación hacia la desincrustación) que chocaría con el resto de esferas sociales y con la tendencia históricamente mayoritaria de la incrustación de los sistemas económicos en los entramados socio-institucionales del conjunto de la sociedad.

Al igual que para Polanyi, para la sociología de la empresarialidad étnica resulta cuestionable el papel que las ciencias sociales han atribuido convencionalmente al mercado (autorregulador) como principio explicativo fundamental de los fenómenos económicos y, de forma más general, de la dinámica de las sociedades contemporáneas. La economía «convencional» (y no pocos representantes de la teoría social), habría efectuado una lectura «economicista» de la dinámica de las sociedades modernas, haciéndola depender, exclusiva o principalmente, de los vaivenes de la dinámica económica (del mercado). Este tipo de aproximaciones, desde el punto de vista de la sociología de la empresarialidad étnica, resultaría insostenible y claramente reduccionista, tal y como habría evidenciado la sistemática presencia (e incidencia) de lo social en los fenómenos económicos: implicación de los marcos normativos y de sentido de los agentes económicos, de sus valores y universos culturales, de las distintas luchas de poder, de la intervención más o menos explícita de diferentes instituciones políticas y sociales, etc.

La presencia de lo normativo y de lo valorativo en lo económico -muy evidente en los fenómenos ligados a la empresarialidad étnica- mostraría que el mercado (una institución social e históricamente circunscrita) no puede explicar por sí mismo la dinámica económica, mucho menos la dinámica general de la sociedad. Al contrario, la esfera económica, como ya señalamos, se encontraba incrustada en un entramado social, político e institucional más amplio del cual no se podía aislar. La tozuda resistencia de lo normativo y de lo regulativo -propios de lo social y de lo político- a desalojar el ámbito de lo económico mostraría de forma nítida, además, el reduccionismo explicativo del paradigma «economicista» presente en las ciencias sociales y, lo que es más importante, el fracaso del proyecto político «neoliberal» de sometimiento y subordinación del conjunto de lo social a la lógica de mercado. El fracaso de la tentativa de evacuar del sistema económico todo principio de regulación externa (social, política) y todo principio de incrustación. En definitiva, el fracaso del proyecto de «aniquilación» y «disolución» de la sociedad (y de lo político) en la lógica del mercado (Polanyi, 1989).

Nos encontramos, por lo tanto, con que la sociología de la empresarialidad étnica va a dar por buena finalmente, la dualización de las formaciones sociales contemporáneas en, al menos, dos esferas: lo económico y lo social (en caso contrario carecería de sentido hablar en términos de incrustación de una esfera en 
otra). En ningún momento va a poner en duda la definición de lo económico como una esfera de carácter sustantivo o como un ámbito específico de la sociedad equiparable al mercado (autorregulado internamente o, lo que es lo mismo, social y políticamente «desregulado»). En ningún momento se va a poner en duda la contraposición radical existente entre esta esfera supuestamente desregulada de la economía (el mercado) y la esfera de la regulación y de la normatividad por antonomasia: la esfera de lo social (y su institucionalización en lo político). Si, como la sociología económica habría demostrado, podemos detectar en «lo económico» aspectos normativos y principios de regulación, estos procederán, necesariamente, de la esfera de lo social (en sus distintas formulaciones institucionales, estatales o no). Toda regulación sería considerada, implícitamente, como una regulación contra el mercado. La sociedad (la norma) contra la economía (el mercado).

Lo único que la sociología de la empresarialidad étnica va a cuestionar, por lo tanto, es que se pretenda dar cuenta de la dinámica de las formaciones sociales contemporáneas exclusivamente desde el ámbito de lo económico, es decir, desde la lógica del mercado, estando éste como está incrustado en lo social. De este modo, como ocurría con el proceder tipológico más arriba señalado, nos encontramos con que la «reformulación» del debate llevada a cabo desde las nuevas aproximaciones de la sociología de la empresarialidad étnica, más que redefinir el objeto de estudio en otros términos (cuestionando, por ejemplo, esta dudosa equiparación de economía y mercado o su contraposición a lo social), se va a limitar a añadir nuevos «contextos» con el objetivo de ganar en «complejidad». La «economía» seguirá siendo una esfera sustantiva identificada con el mercado autorregulado, residiendo ahora el desafío (y la aportación de los sociólogos) en incorporar a los análisis (y a los modelos de organización colectiva) la esfera de «lo social» (lo normativo, lo cualitativo, lo valorativo, etc.).

En lo que a nosotros se refiere, no nos cabe la menor duda de que, en efecto, el mercado es incapaz de dar cuenta de la dinámica de las formaciones sociales contemporáneas. De hecho, podemos afirmar sin miedo que es incapaz, incluso, de dar cuenta de la propia dinámica «económica». No obstante, lo que convendría someter a discusión es, precisamente, la rápida identificación de capitalismo y mercado, así como la posterior contraposición de lo económico a lo normativo y, en definitiva, a lo social. Vistos, por ejemplo, los hallazgos historiográficos sobre el papel desempeñado por el Estado y lo normativo en la conformación histórica de formaciones sociales capitalistas en Occidente (Anderson, 1984; Braudel, 1985; Polanyi, 1989; Castel, 2004), o los análisis sobre la cohabitación de planificación y mercado en los mecanismos contemporáneos del salariado (Naville, 1970; Alaluf, 1986; Pillon y Vatin, 2007; Rolle, 2009) itiene sentido seguir considerando los principios de regulación como elementos «excepcionales» y «externos» a la lógica económica (social) capitalista? ¿Debemos seguir insistiendo en contraponer norma a mercado como dos principios antitéticos?

Tanto la identificación de la «economía» capitalista con una esfera sustantiva e institucionalizada de la sociedad (el mercado), como la consideración más 
universalista de la economía como ámbito orientado, en toda colectividad humana, al aprovisionamiento de los medios y las utilidades con los que satisfacer las necesidades ¿no nos hacen perder de vista la especificidad histórica de las formaciones sociales capitalistas? Polanyi (2004) trató de analizar los cambios en el lugar ocupado por la economía en la sociedad a lo largo de la historia y de las culturas, pero ¿y si la economía en las formaciones sociales capitalistas no ocupasen «un lugar» en particular? ¿Y si no se tratase de una esfera sustantiva opuesta a lo social? ¿Y si nos encontrásemos con que, por primera vez en la historia de las colectividades humanas, la «economía» constituyera más bien un mecanismo, un método, una gramática con la que producir, conjugar y ordenar (más que destruir o aniquilar) la sociedad?

La hipótesis que creemos necesario formular para abordar este debate en términos explicativamente más productivos sostiene que, a diferencia de otro tipo de sociedades, en las formaciones sociales capitalistas, economía y sociedad son, en realidad, dos modos de nombrar una única y misma realidad. Es decir, que regulación (norma) y mercado no constituyen dos principios contrapuestos, sino dos mecanismos de movilización y asignación de recursos que coexisten en las sociedades capitalistas contemporáneas (al igual que coexistieron en el denominado socialismo real). Si esto es así, es cierto que no podremos reconocer automáticamente en la regulación social, comunitaria, política o administrada de los intercambios ninguna potencialidad emancipadora (sea ésta formulada en términos reformistas o revolucionarios), ningún más allá o más acá de las relaciones constitutivas de las formaciones sociales capitalistas (relaciones difícilmente asimilables a ninguna lógica de mercado). A cambio de esta orfandad, la verdadera «liberación» residirá en habernos desprendido, por fin, de la ilusión de construir un capitalismo «de rostro humano», un nuevo capitalismo paria o la enésima república de artesanos.

\section{Bibliografía}

Alaluf, M. (1986). Le temps du labeur. Formation, emploi et qualification en sociologie du travail. Bruselas: Éditions de l’ULB.

Alaluf, M. (1993). El empleo, una relación social inestable. En F. Michon y D. Segrestin (Coord.), El empleo, la empresa y la sociedad (pp. 305-312). Madrid: Ministerio de Trabajo.

Aldrich, H. y Waldinger, R. (1990). Ethnicity and Entrepreneurship. Annual Review of Sociology. Vol. 16: 111-135. doi: 10.1146/annurev.so.16.080190.000551

Anderson, P. (1984). El Estado absolutista. Madrid: Siglo XXI.

Aramburu, M. (2.002). Los comercios de inmigrantes en Barcelona y la recomposición 
del "inmigrante" como categoría social. Scripta Nova, Vol. VI, Núm. 108, 105-132.

Arjona, A. (2006). Los col6ores del escaparate. Emprendedores inmigrados en Almería. Barcelona: Icaria.

Arjona, A. y Checa, J.C. (2006). Empresariado extracomunitario en Almería: estructuras de oportunidad, características de grupo y estrategias étnicas. Revista Española de Investigaciones Sociológicas, Núm. 115, 297-317

Beltrán, J.; Oso, L. y Ribas, N. (coords.) (2006). Empresariado étnico en España. Madrid: Fundació CIDOB/Observatorio Permanente de la Inmigración.

Bonacich, E. (1973). A theory of middleman minorities. American Sociological Review, Vol. 38, 583-594.

Bonacich, E. (1975). Small Business and Japanese American Ethnic Solidarity. Amerasia Journal, Vol. 3, Núm. 1, 96-112.

Bonacich, E. y Modell, J. (1980). The Economic Basis of Ethnic Solidarity in the Japanese American Community. Berkeley: University of California Press.

Braudel, F. (1985). La dinámica del capitalismo. Madrid: Alianza.

Castel, R. (2004). La metamorfosis de la cuestión social. Una crónica del salariado. Buenos Aires: Paidós.

Cavalcanti, L. (2008). Negocios étnicos: importación y repercusiones de una categoría. En E. Santamaría (ed.), Los retos epistemológicos de las migraciones transnacionales (pp. 217-236). Barcelona: Anthropos.

Dallalfar, A. (1994). Iranian Women as Immigrant Entrepreneurs. Gender and Society, Vol. 8, Núm. 4: 541-561. doi: 10.1177/089124394008004005

Der-Martirosian, C.; Sabagh, G. y Bozorgmehr, M. (1993). Subethnicity: Armenians in Los Angeles. En I. Light y P. Bhachu (Ed.), Immigration and Entrepreneurship. Culture, Capital and Ethnic Networks (pp. 243-258). New Brunswick: Transaction Publishers.

Forment, C. (1989). Political practice and the rise of an ethnic enclave. The Cuban American Case, 1959-1979. Theory and Society, Vol. 18, 47-81. doi: 10.1007/BF00160688

Freeman, G. y Ögelman, N. (2000). State Regulatory Regimes and Immigrants' Informal Economic Activity. En J. Rath (Ed.), Immigrant Businesses. The 
Economic, Political and Social Environment (pp. 107-123). Londres: Palgrave MacMillan Press.

Gap Min, P. (1996). Caught in the Middle. Koreans Communities in New York and Los Angeles. Berkeley: University of California Press.

Gilbertson, G. (1995). Women's Labor and Enclave Employment: The Case of Dominican and Colombian Women in New York City. International Migration Review, Vol. 29, Núm. 3, 657-670.

Godelier, M. (1996). L'enigme du don. París: Fayard.

Granovetter, M. (1985). Economic Action and Social Structure: The Problem of Embeddedness. American Journal of Sociology, Vol. 91, 481-510. doi: $10.1086 / 228311$

Guarnizo, L. (2003). The Economics of Transnational Living. International Migration Review, Vol. 37, Núm. 3, 666-699. doi: 10.1111/j.17477379.2003.tb00154.x

Hillmann, F. (1999). A Look at the "Hidden Side": Turkish Women in Berlin's Ethnic Labour Market. International Journal of Urban and Regional Research, Vol. 23, Núm. 2, 267-282. doi: 10.1111/1468-2427.00195

Hobsbawm, E. (1988). En torno a los orígenes de la revolución industrial. Madrid: Siglo XXI.

Jones, T.; Barret, G. y McEvoy, D. (2000). Market Potential as a Decisive Influence on the Performance of Ethnic Minority Business. En J. Rath (Ed.), Immigrant Businesses. The Economic, Political and Social Environment (pp. 37-53). Londres: Palgrave MacMillan Press.

Josephides, S. (1988). Honour, Family and Work: Greek Cypriot Women Before and After Migration. En S. Westwood y P. Bhachu (Eds.), Enterprising Women. Ethnicity, Economy and Gender Relations (pp. 34-57). Londres: Routledge.

Kloosterman, R. (2000). Immigrant Entrepreneurship and the Institutional Context: A Theoretical Exploration. En J. Rath (Ed.), Immigrant Businesses. The Economic, Political and Social Environment (pp. 90-106). Londres: Palgrave MacMillan Press.

Kloosterman, R.; Van Der Leun, J. y Rath, J. (1999). Mixed Embeddedness: (In)formal Economic Activities and Immigrant Bussiness in the Netherlands. Journal of Urban and Regional Research, Vol. 23, Núm. 2, 252-266. doi: $10.1111 / 1468-2427.00194$ 
Kloosterman, R. y Rath, J. (2001). Immigrant entrepreneurs in advanced economies: mixed embeddedness further explored. Journal of Ethnic and Migration Studies, Vol. 27, Núm. 2: 189-201. doi: 10.1080/13691830020041561

Kwok Bun, C. y Jin Hui, O. (1995). The many faces of immigrant entrepreneurship. En R. Cohen (Ed.), The Cambridge Survey of World Migration (pp. 523-531). Cambridge: Cambridge University Press.

Light, I. (1972). Ethnic Enterprise in America. Business and Welfare Among Chinese, Japanese and Blacks. Berkeley: University of California Press.

Light, I. y Bonacich, E. (1988). Immigrant Entrepreneurs. Koreans in Los Angeles 1965-1982. Los Angeles: University of California Press.

Light, I. y Rosenstein, C. (1995a). Race, Ethnicity and Entrepreneurship in Urban America. Nueva York: Aldine De Gruyter.

Light, I. y Rosenstein, C. (1995b). Expanding the Interaction Theory of Entrepreneurship. En A. Portes (Ed.), The Economic Sociology of Immigration. Essays on Networks, Ethnicity and Entrepreneurship (pp. 166-212). Nueva York: Russell Sage Foundation.

Light, I. y Gold, S. (2000). Ethnic Economies. San Diego: Academic Press.

López-Castellano, F.; García, F. y Aboussi, M. (Comps.) (2012). Empresariado inmigrante. Instituciones y desarrollo. Granada: Comares.

López-Sala, A. (2007). Migración internacional, vínculos transnacionales y economía étnica. El caso de la comunidad indostánica en Canarias. Revista CIDOB d'Afers Internacionals, Vol. 78, 97-120.

Mauss, M. (1950). Sociologie et Anthropologie. París: Presses Universitaires de France.

Moreras, J. (2005). Iniciativas comerciales inmigrantes en un contexto urbano en transformación: el caso de Ciutat Vella (Barcelona). En J. Beltrán; L. Oso y N. Ribas (Coords.) Empresariado étnico en España (pp. 129-154). Madrid: Fundació CIDOB/Observatorio Permanente de la Inmigración.

Morokvasic, M. (1988). Entrepreneurship, ethnicity and gender. En S. Westwood y P. Bhachu (Eds.), Enterprising Women. Ethnicity, Economy and Gender Relations (pp. 20-33). Londres: Routledge. 
Naville, P. (1970). Le nouveau léviathan, II. Le salaire socialiste. Les rapports de production. París: Éditions Anthropos.

Nederveen Pieterse, J. (2003). Social Capital and Migration. Beyond ethnic economies. Ethnicities, Vol. 31, Núm. 1 29-58. doi: $10.1177 / 1468796803003001785$

Oso, L. y Villares, M. (2008). Latinoamericanos empresarios en España: una estrategia de movilidad ocupacional. En G. Herrera y J. Ramírez (ed.) América Latina migrante: Estado, familias, identidades (pp. 159-175). Quito: Flacso.

Pessar, P. (1995). The Elusive Enclave: Ethnicity, Class and Nationality among Latino Entrepreneurs in Greater Washington DC. Human Organization, Vol. 54, Núm. 4, 383-392.

Pillon, T. y Vatin, F. (2007). La question salariale: actualité d'un vieux problème. En F. Vatin (Dir.), Le salariat. Théorie, histoire et formes (pp. 29-48). París: La Dispute.

Pizarro, N. (1979). El sujeto y los valores: la sociología de la acción de Alain Touraine. Revista Española de Investigaciones Sociológicas, Vol. 5, 37-53.

Polanyi, K. (1989). La Gran Transformación. Madrid: La Piqueta.

Polanyi, K. (2004) El sistema económico como proceso institucionalizado. En P. Moreno (Comp.), Entre las gracias y el molino satánico: lecturas de antropología económica (pp. 233-259). Madrid: UNED.

Portes, A. (1981). Modes of Structural Incorporation and Present Theories of Labor Immigration. En M. Kritz, Ch. Keely y S. Tomasi (Eds.), Global Trends in Migration (pp. 279-297). Nueva York: Center For Migration Studies.

Portes, A. (Ed.) (1995). The Economic Sociology of Immigration. Essays on Networks, Ethnicity and Entrepreneurship. Nueva York: Russell Sage Foundation.

Portes, A. (2000). The Two Meannings of Social Capital. Sociological Forum, Vol. 15, Núm. 1: 1-12. doi: 10.1023/A:1007537902813

Portes, A. (2010). Economic Sociology. A Systematic Inquery. Princeton: Princeton University Press

Portes, A. y Bach, R. (1985). Latin Journey. Cuban and Mexican Immigrants in the U.S. Berkeley: University of California Press.

Portes, A. y Stepick, A. (1985). Unwelcome immigrants: the labor market experiences of 1980 (Mariel) Cuban and Haitian refugees in South Florida. 
American Sociological Review, Vol. 50, Núm. 4, 493-514.

Portes, A. y Stepick, A. (1993). City on the Edge. The Transformation of Miami. Berkeley: University of California Press.

Portes, A. y Jensen, L. (1989). The enclave and the entrants: patterns of ethnic enterprise in Miami before and after Mariel. American Sociological Review, Vol. 54, Núm. 6, 929-949.

Portes, A. y Sensenbrenner, J. (1993). Embeddedness and Immigration: Notes on the Social Determinants of Economic Action. American Journal of Sociology, Vol. 98, Núm. 6, 1320-1350.

Portes, A. y Zhou, M. (1992). Gaining the upper hand: economic mobility among immigrant and domestic minorities. Ethnic and Racial Studies, Vol. 15, Núm. 4: 491-522. doi: 10.1080/01419870.1992.9993761

Portes, A.; Guarnizo, L. y Haller, W. (2002). Transnacional Entrepreneurs: An Alternative Form of Immigrant Economic Adaptation. American Sociological Review, Vol. 67, Núm. 2, 278-298.

Postone, M. (2006). Tiempo, trabajo y dominación social. Madrid: Marcial Pons.

Rath, J. (2000). Introduction: Immigrant Businesses and their Economic, PoliticoInstitutional and Social Environment. En J. Rath (Ed.), Immigrant Businesses. The Economic, Political and Social Environment (pp. 1-19). Londres: Palgrave MacMillan Press.

Rath, J. y Oliveira, C. (2008). Introduction to the Special Issue on Immigrant Entrepreneurship. Migraçoes Journal, Vol. 3, 11-27

Riesco-Sanz, A. (2003). Enclaves y economías étnicos desde la perspectiva de las relaciones salariales. Cuadernos de Relaciones Laborales, Vol. 21, Núm. 2, 103-125.

Riesco-Sanz, A. (2010). Inmigración y trabajo por cuenta propia. "Economías inmigrantes» en Lavapiés (Madrid). Madrid: Universidad Complutense de Madrid (en línea). http://eprints.ucm.es/11412/

Rolle, P. (1988). Travail et salariat. Bilan du sociologie $d u$ travail. Grenoble: Presses Universitaires de Grenoble.

Rolle, P. (2005). El trabajo y su medida. En García López et al. (Comp.), Lo que el trabajo esconde. Materiales para un replanteamiento del análisis sobre el trabajo (pp. 119-130). Madrid: Traficantes de Sueños. 
Rolle, P. (2009). De la revolución del trabajo al trabajo revolucionado. Madrid: Traficantes de Sueños.

Sanders, J. y Nee, V. (1987a). Limits of ethnic solidarity in the enclave economy. American Sociological Review, Vol. 52, Núm. 6, 745-767.

Sanders, J. y Nee, V. (1987b). On testing the enclave-economy hypothesis. American Sociological Review, Vol. 52, Núm. 6, 771-773

Sanders, J. y Nee, V. (1992). Problems in Resolving the Enclave Economy Debate. American Sociological Review, Vol. 57, Núm. 3, 415-418.

Serra, P. (2006). El comercio étnico en el distrito de Ciutat Vella de Barcelona. Barcelona: Fundació "La Caixa".

Simmel, G. (1977). Sociología. Estudio sobre las formas de socialización. Madrid: Revista de Occidente.

Solé, C.; Parella, S. y Cavalcanti, L. (2007). El empresariado inmigrante en España. Barcelona: Fundación La Caixa.

Solé, C.; Parella, S. y Alarcón, A. (2009). El autoempleo de las trabajadoras inmigradas. ¿Una alternativa a la discriminación laboral? Cuadernos de Relaciones Laborales, Vol. 27, Núm. 1, 171-200

Sombart, W. (2001). The Jews and Modern Capitalism. Kitchener: Batoche Books.

Waldinger, R. (1986a). Immigrant Entreprise: A Critique and Reformulation. Theory and Society, Vol. 15, Núm. 1-2: 249-285. doi: 10.1007/BF00156934

Waldinger, R. (1986b). Through the Eye of the Needle. Immigrants and Enterprise in New York's Garment Trade. Nueva York: New York University Press

Waldinger, R. (1989). Structural opportunity or Ethnic Advantage? Immigrant Business Development in New York? International Migration Review, Vol. 23, Núm. 1, 48-72.

Waldinger, R.; Aldrich, H. y Ward, R. (1990). Opportunities, Group Characteristics and Strategies. En R. Waldinger, H. Aldrich y R. Ward (Eds.), Ethnic Entrepreneurs: Immigrant Business in Industrial Societies (pp. 9-48). Newbury Park: Sage. 
Weber, M. (1974). Historia Económica General. México D.F.: FCE.

Weber, M. (1993a). La ética protestante y el espiritu del capitalismo. Barcelona: Península.

Weber, M. (1993b). Economía y Sociedad. México DF: FCE.

Wilson, K. y Portes, A. (1980). Immigrant enclaves: An analysis of the labor market experiences of cubans in Miami. American Journal of Sociology, Vol. 86, Núm. 2, 295-319.

Wilson, K. y Martin, A. (1982). Ethnic Enclaves: A Comparison of the Cuban and Black Economies in Miami. American Journal of Sociology, Vol. 88, Núm. 1, 135-160.

Young Kim, D. (1999). Beyond co-ethnic solidarity: Mexican and Ecuadorean employment in Korean-owned businesses in New York City. Ethnic and Racial Studies, Vol. 22, Núm. 3: 581-605. doi: 10.1080/014198799329431

Zhou, M. (1992). Chinatown. The Socioeconomic Potential of an Urban Enclave. Philadelphia: Temple University Press.

Zhou, M. y Logan, J. (1989). Returns on Human Capital in Ethnic Enclaves: New York City's Chinatown. American Sociological Review, Vol. 54, Núm. 5, 809-820. 